\title{
Comparison of spectra of materials measured by Time Domain and Fourier Transform Spectroscopy in Terahertz range
}

\author{
Norbert Palka ${ }^{* 1}$, Mieczyslaw Szustakowski ${ }^{1}$, Tomasz Trzcinski ${ }^{1}$, and Malgorzata Mozdzonek ${ }^{2}$ \\ ${ }^{I}$ Institute of Optoelectronics, Military University of Technology, Kaliskiego 2, 00-908 Warszawa, \\ ${ }^{2}$ Institute of Electronic Materials Technology, Wolczynska 133, 01-919 Warszawa
}

Received April 12, 2011; accepted April 15, 2011; published June 30, 2011

\begin{abstract}
Absorption spectra in $\mathrm{THz}$ range of commonly used explosive materials - Hexogen, Penthrite, Octogen and Sugar are presented. Explosives were prepared as pellets, where Teflon was applied as a matrix material. The measurements were carried out by means of Time Domain Spectroscopy and compared to spectra obtained by Fourier Transform Spectroscopy. Good conformity is observed. We also present spectra of cotton and paper as well as RDX hidden under cotton.
\end{abstract}

The development and implementation of terahertz $(0.1$ $10 \mathrm{THz}$ ) technology in the security area is connected with unique features of terahertz radiation. The frequency of terahertz radiation corresponds well with the frequency of a normal mode (vibrational and rotational) of oscillating molecules of chemical compounds at room temperature and acoustic vibrations of molecules. Accordingly, chemical compounds of dangerous materials have specific absorption and emission spectra in the terahertz range, which allows us to identify them. So, many explosives (e.g., Hexogen, Penthrite, Trinitrotoluene, Octogen) and drugs have characteristic transmission/reflection features in the $\mathrm{THz}$ range that could be distinguishable from other common materials like clothes, human skin or metal materials [1].

Moreover, terahertz waves penetrate many materials apart from metals or polar mediums (like water). Therefore, terahertz radiation can scan through packing, clothes, shoes, bags and is suitable for screening baggage and primarily for looking for materials hidden on human body under covering. Such advantages are of great importance for security and defense related applications.

The spectra of pure explosives in the $\mathrm{THz}$ range have been measured by many groups [2-5]. However, the spectra of the same material can differ because of a different sample preparation process. One should take into account the size of particles and the material which is used to dissolve explosives (usually Teflon or HDPE). Moreover, some differences in spectra are observed because of different measurement techniques (Time Domain Spectroscopy versus Fourier Transform Infrared Spectroscopy) and water vapor influences.

\footnotetext{
*npalka@wat.edu.pl
}

In this paper we report on measurements of absorption spectra of Hexogen (RDX), Penthrite (PETN), Octogen (HMX), and Sugar (Sucrose) in the range 0.1-3.5THz. The measurements were carried out by means of Time Domain Spectroscopy and compared to spectra obtained by Fourier Transform Infrared Spectroscopy. Only small discrepancies are observed. The obtained characteristics can be used to create a data base of spectra of explosive materials.

For the purpose of our measurements we manufactured samples of three commonly used explosives: Heksogen (RDX, Cyclotrimethylene trinitramine), Pentrite (PETN, Pentaerythritol tetranitrate) and Octogen (HMX, Cyclotetramethylene tetranitramine), which have characteristic peaks in the $\mathrm{THz}$ range. Moreover, we decided to measure Sugar, which can be also used as an explosive. The specified materials have high attenuation, so we decided to mix them with Politetrafluoroetylen (PTFE, Teflon) powder, which is highly transparent in the $\mathrm{THz}$ range. The explosives were ground using a mortar and pestle to reduce the particle size and to avoid scattering loss [2]. Next, the explosives were mixed with PTFE powder. The mixing ratio was $10 \%$ (weight of RDX versus total weight of mixed samples). Afterwards, the sample was pressed into pellets (weight 400mg, $1.4 \mathrm{~mm}$ in thickness and $13 \mathrm{~mm}$ in diameter) directly using a hydraulic press. As a result, we obtained the sample which consisted of $40 \mathrm{mg}$ of explosives. As a reference pellet, a pellet made of pure Teflon with the same dimension and weight was prepared.

There are two main techniques used for the measurement of transmission spectra in the $\mathrm{THz}$ range: Fourier Transform Infrared Spectroscopy (FTIR) and Time Domain Spectroscopy (TDS). In both cases, we measured spectra of the material pellet and the reference pellet $\left(T_{R}\right)$. Next, the transmission properties of the explosives can be presented as Absorbance $(A)$, which is defined as:

$$
A=-\log _{10}\left(T_{M} / T_{R}\right)
$$


A typical far-infrared Fourier transform spectroscopy setup consists of a source - an incoherent high-pressure mercury arc lamp useful for the $\mathrm{THz}$ range and a piroelectric (DTGS) or a bolometer detector and a scanning Michelson interferometer (a far-IR beam splitter, mirrors, a motorized delay line), samples, and a data acquisition system. Because of the short coherence of the source, during the scanning of the mirror an interferogram is recorded as a function of mirror position. Next, the data should be processed by means of the Fourier transform to obtain the spectrum (Fig. 1).

In our case, the samples were measured by means of spectrometer FTIR Bruker 113 in the range $0.5-6 \mathrm{THz}$ with resolution $1 \mathrm{~cm}^{-1}$ in vacuum ( $3 \mathrm{mbar}$ ) and temperature $298 \mathrm{~K}$. Figure 4 presents Absorbance $(A)$ of the following explosives: RDX, PETN, HMX and Sugar.

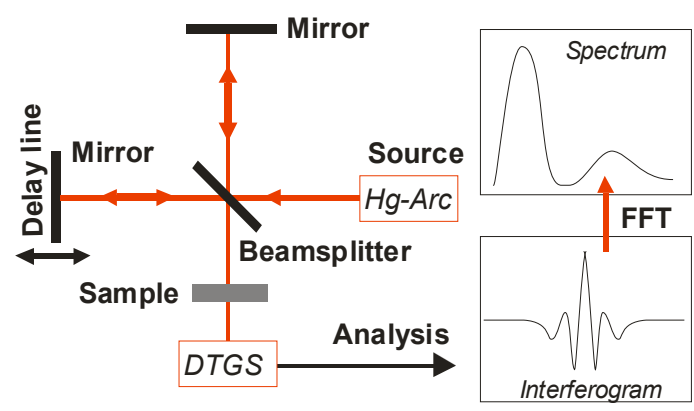

Fig. 1. Fourier Transform Infrared Spectroscopy setup.

A Time Domain Spectrometry setup from EKSPLA was applied (Fig. 2). It is based on pulses generated by an $800 \mathrm{~nm}$ femtosecond laser (Fusion20, Femtolaser, a pulse duration of $20 \mathrm{fs}$, repetition rate of $80 \mathrm{MHz}$, and average power of $200 \mathrm{~mW}$ ). The laser beam was split into a pump and probe and directed through a system of mirrors to an emitter and a detector. Low-temperature-grown GaAs dipole antennas with a gap distance of about $6 \mu \mathrm{m}$ were applied as the emitter and the detector. The pump beam was focused on the biased emitter antenna to generate $\mathrm{THz}$ pulses through a photoconductive phenomenon. The emitted $\mathrm{THz}$ pulses were collimated by a Silicon lens. The pellets were placed in the middle of the distance between the emitter and the detector, perpendicular to the incident $\mathrm{THz}$ beam. The transmitted $\mathrm{THz}$ beam was detected by means of the detector antenna gated by the laser probe beam and a mechanical delay line. A lock-in amplifier and LabView-based software were used to collect and process data. The system was purged with dry nitrogen gas to eliminate water vapor.

Figure 3 a presents two $\mathrm{THz}$ impulses - measured with a pure Teflon sample (reference pellet) and with a pellet which consists of a mixture of Teflon and an explosive (the material pellet). The impulses were apodized by means of Blackman-Harris 3 term filter. Figure $3 \mathrm{~b}$ shows the $\mathrm{THz}$ spectra, ranging between 0.1 and $5 \mathrm{THz}$, obtained by applying Fast Fourier Transform (FFT) to the $\mathrm{THz}$ impulses. The useful range is from 0.1 up to $3.5 \mathrm{THz}$.

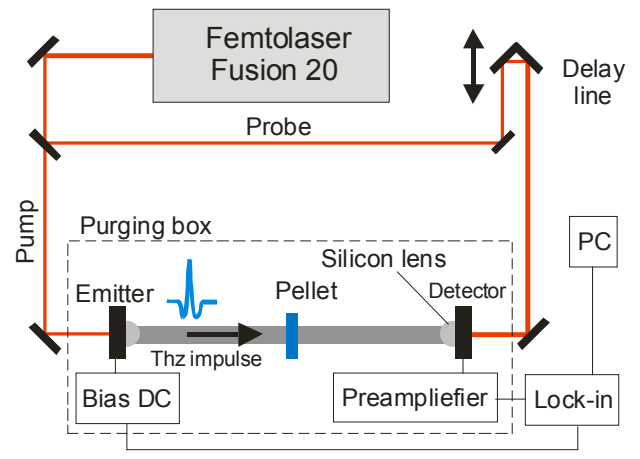

Fig. 2. Time Domain Spectrometry setup
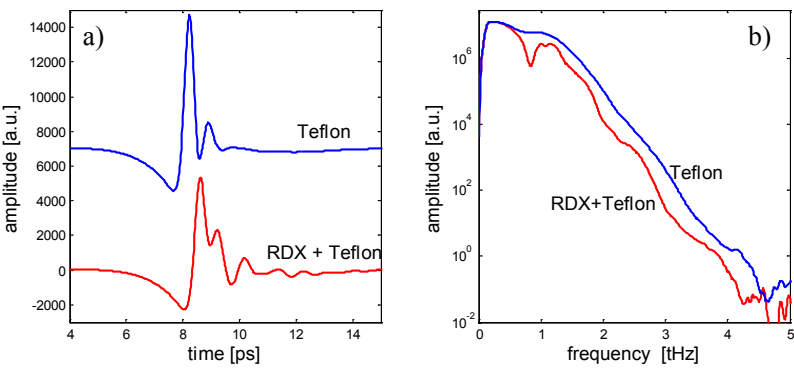

Fig. 3. THz impulses measured for Teflon and Teflon-RDX pellets offset added for clarity (a) and FFT of the impulses (b).

It is clearly seen in Fig. 3 that explosives in the pellets change the shape of the impulses as well as spectra (FFT modules). A time shift between impulses in Fig. $3 \mathrm{a}$ is connected with the fact that samples with explosives have slightly bigger refractive indices, which increases the optical path inside the pellets. This shift can also results from the fact that the pellets can differ in thickness because of inaccurate preparation process.

Figure 4 presents the absorbance of the following explosives: RDX, PETN, HMX and Sugar measured by TDS and FTIR.

The spectra measured by the two methods are in good conformity, taking into account the shape of the spectra and positions of characteristic peaks. Some discrepancies are connected with water vapor absorption and reflections in the TDS setup, which will be further optimized. The values of absorbance below $0.5 \mathrm{THz}$ in FTIR are not accurate because this range is close to the limit of the system. On the other hand, values above $3.5 \mathrm{THz}$ in the TDS method suffer from the bandwidth limit of our experimental system. The obtained main peaks of absorbance remain in good agreement with the results of other groups [2-5]. 

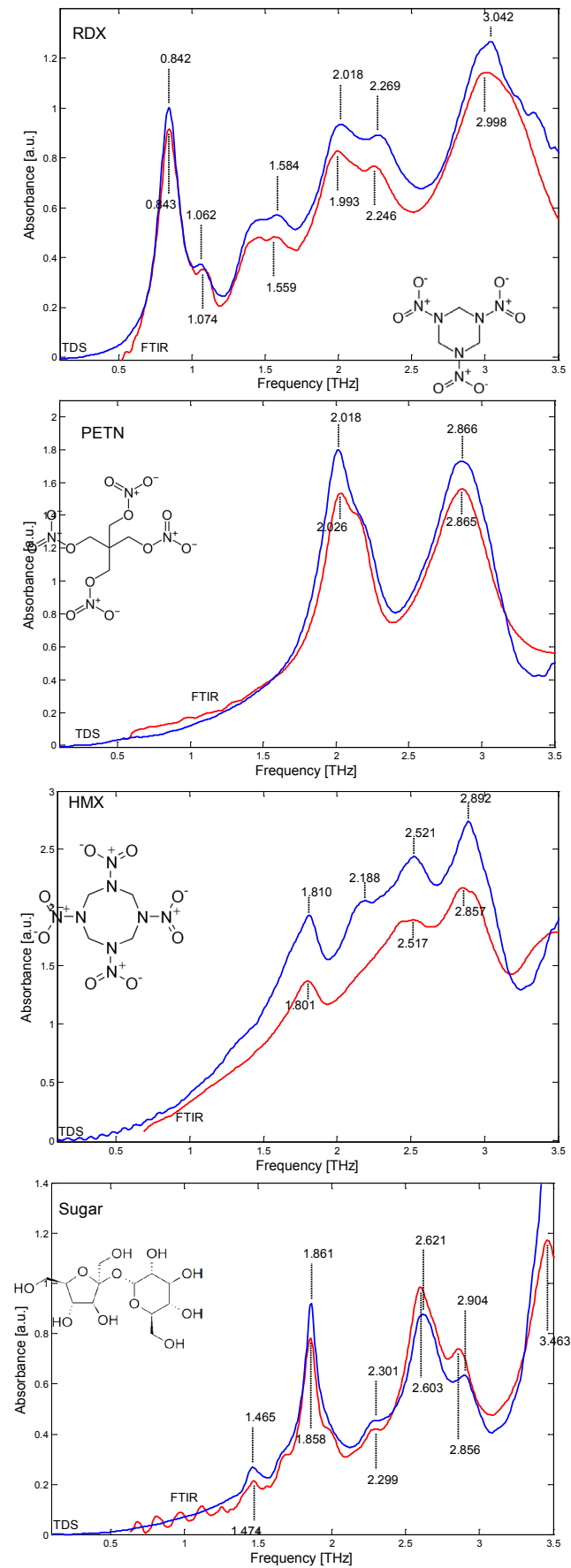

Fig. 4. Absorbance of RDX, PETN, HMX and Sugar measured by TDS and FTIR.

As the $\mathrm{THz}$ spectroscopy is perceived as a perspective tool for detection of hidden explosives, we also measured and compared the spectra of cotton (a piece of t-shirt) and a sheet of paper $\left(80 \mathrm{~g} / \mathrm{m}^{3}\right)$ - commonly used packaging materials (Fig. 5). Next, we also show the spectrum of RDX hidden under the piece of cotton (Fig. 6).

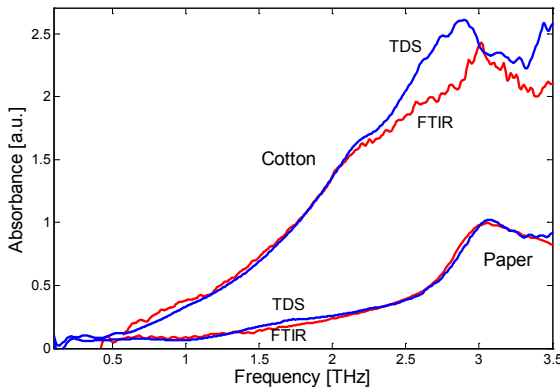

Fig. 5. Absorbance of Paper and Cotton measured by TDS and FTIR.

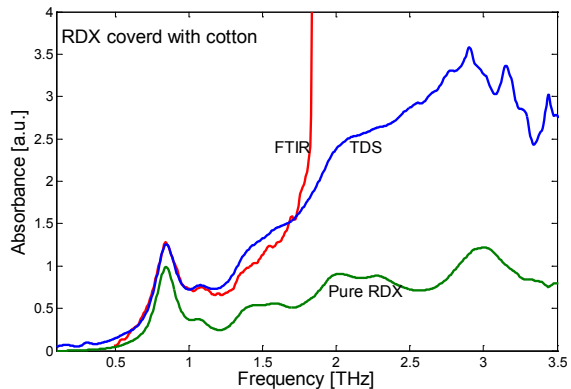

Fig. 6. Absorbance of RDX covered under Cotton (TDS and FTIR).

The results reveal that packaging materials are quite transparent in the $0.1-3 \mathrm{THz}$ range and their spectra measured by both methods stay in conformity.

For the RDX sample cover with cotton, both methods give the same results up to $1.7 \mathrm{THz}$. Above $1.7 \mathrm{THz}$ for FTIR spectrometer attenuation of the sample is too high to be measured while TDS can be easily measured up to $3 \mathrm{THz}$ with good performance. Typical features of RDX can be identified: $0.84 \mathrm{THz}, 1.06 \mathrm{THz}$, and $1.58 \mathrm{THz}$, which indicates that packaging materials do not present too much distortion and shift of absorption peak locations.

The paper is co-financed by the Polish Ministry of Science and Education under PRUE/31-436-2008-WAT.

\section{References}

[1] L.Yun-Shik, Principles of Terahertz Science and Technology (Springer 2008).

[2] J. Chen, Y. Chen, H. Zhao, G. Bastiaans, X.-C. Zhang, Opt. Exp. 15, 12060 (2007).

[3] M.R. Leahy-Hoppa, M.J. Fitch, X. Zheng, L.M. Hayden, R. Osiander, Chemical Phys. Lett. 434, 227 (2007).

[4] D. J. Cook, B. K. Decker, M.G. Allen, Optical Terahertz Science and Technology (Orlando 2005).

[5] D. J. Cook, M. G. Allen, B. K Decker, R.T. Wainner, J.M. Hensley, H.S. Kindle, Joint 30th International Conference on Infrared and Millimiter Waves and 13ht International Conference on Terahertz Electronics, Williamsburg, VA, 19-23 September (2005). 